\title{
Article
}

\section{Uniqueness of Abel's Integral Equations of the Second Kind with Variable Coefficients}

\author{
Chenkuan Li * (D) and Joshua Beaudin (D) \\ Department of Mathematics and Computer Science, Brandon University, Brandon, MB R7A 6A9, Canada; \\ beaudijd31@brandonu.ca \\ * Correspondence: lic@brandonu.ca
}

check for

updates

Citation: Li, C.; Beaudin, J.

Uniqueness of Abel's Integral

Equations of the Second Kind with

Variable Coefficients. Symmetry 2021,

13, 1064. https://doi.org/10.3390/

sym13061064

Academic Editors: Francisco

Martínez González and Mohammed KA Kaabar

Received: 30 May 2021

Accepted: 11 June 2021

Published: 13 June 2021

Publisher's Note: MDPI stays neutral with regard to jurisdictional claims in published maps and institutional affiliations.

Copyright: (c) 2021 by the authors. Licensee MDPI, Basel, Switzerland. This article is an open access article distributed under the terms and conditions of the Creative Commons Attribution (CC BY) license (https:// creativecommons.org/licenses/by/ $4.0 /)$.

\begin{abstract}
This paper studies the uniqueness of the solutions of several of Abel's integral equations of the second kind with variable coefficients as well as an in-symmetry system in Banach spaces $L(\Omega)$ and $L(\Omega) \times L(\Omega)$, respectively. The results derived are new and original, and can be applied to solve the generalized Abel's integral equations and obtain convergent series as solutions. We also provide a few examples to demonstrate the use of our main theorems based on convolutions, the gamma function and the Mittag-Leffler function.
\end{abstract}

Keywords: partial Riemann-Liouville fractional integral; Babenko's approach; Banach fixed point theorem; Mittag-Leffler function; gamma function

\section{Introduction}

$$
\begin{aligned}
& \text { Let } 0<\Omega_{i}<\infty \text { for } i=1,2, \cdots, n \text {, and } \Omega=\left[0, \Omega_{1}\right] \times\left[0, \Omega_{2}\right] \times \cdots \times\left[0, \Omega_{n}\right] \subset R^{n} \text {. Define: } \\
& \qquad L(\Omega)=\left\{u \mid u \text { is Lebesgue integrable on } \Omega \text { and }\|u\|=\int_{\Omega}|u(x)| d x<\infty\right\} .
\end{aligned}
$$

Furthermore, the product space $L(\Omega) \times L(\Omega)$ is given by

$$
L(\Omega) \times L(\Omega)=\{(u, v) \mid u, v \text { are Lebesgue integrable on } \Omega \text { and }\|(u, v)\|<\infty\},
$$

where:

$$
\|(u, v)\|=\int_{\Omega}|u(x)| d x+\int_{\Omega}|v(x)| d x .
$$

Clearly, both $L(\Omega)$ and $L(\Omega) \times L(\Omega)$ are Banach spaces.

Let $I_{k}^{\alpha}$ be the partial Riemann-Liouville fractional integral of order $\alpha \in R^{+}$with respect to $x_{k} \in\left[0, \Omega_{k}\right]$, with initial point zero [1]:

$$
\left(I_{k}^{\alpha} u\right)(x)=\frac{1}{\Gamma(\alpha)} \int_{0}^{x_{k}}\left(x_{k}-s\right)^{\alpha-1} u\left(x_{1}, \cdots, x_{k-1}, s, x_{k+1}, \cdots, x_{n}\right) d s
$$

for $k=1,2, \cdots, n$.

In particular:

$$
\left(I_{k}^{0} u\right)(x)=u(x)
$$

Assume that $\lambda_{i j}(x)$ is the Lebesgue integrable and bounded on $\Omega$ for all $i=1,2, \cdots, n \in$ $N$ and $j=1,2, \cdots, m \in N$. In this paper, we begin to construct a unique solution in the space $L(\Omega)$ using Babenko's method and properties of the gamma function for the following generalized Abel's integral equation of the second kind with variable coefficients for $f \in L(\Omega)$ :

$$
u(x)+\sum_{j=1}^{m}\left\{\lambda_{1 j}(x) I_{1}^{\alpha_{1 j}}\right\}\left\{\lambda_{2 j}(x) I_{2}^{\alpha_{2 j}}\right\} \cdots\left\{\lambda_{n j}(x) I_{n}^{\alpha_{n j}}\right\} u(x)=f(x)
$$


where each fractional integral $I_{k}^{\alpha_{k j}}$ carries its own weight function $\lambda_{k j}(x)$, and all $\alpha_{i j} \geq 0$ satisfy a certain condition. Then, we further study the uniqueness of solutions in $L(\Omega)$ for:

$$
u(x)+\sum_{j=1}^{m}\left\{\lambda_{1 j}(x) I_{1}^{\alpha_{1 j}}\right\}\left\{\lambda_{2 j}(x) I_{2}^{\alpha_{2 j}}\right\} \cdots\left\{\lambda_{n j}(x) I_{n}^{\alpha_{n j}}\right\} u(x)=g(x, u(x)),
$$

where $g(x, y)$ is a mapping from $\Omega \times R$ to $R$. Finally, the sufficient conditions are given for the uniqueness of solutions in $L(\Omega) \times L(\Omega)$ to the symmetric system:

$$
\left\{\begin{array}{l}
u(x)+\sum_{j=1}^{m}\left\{\lambda_{1 j}(x) I_{1}^{\alpha_{1 j}}\right\}\left\{\lambda_{2 j}(x) I_{2}^{\alpha_{2 j}}\right\} \cdots\left\{\lambda_{n j}(x) I_{n}^{\alpha_{n j}}\right\} u(x)=g_{1}(x, u(x), v(x)) \\
v(x)+\sum_{j=1}^{m}\left\{\mu_{1 j}(x) I_{1}^{\beta_{1 j}}\right\}\left\{\mu_{2 j}(x) I_{2}^{\beta_{2 j}}\right\} \cdots\left\{\mu_{n j}(x) I_{n}^{\beta_{n j}}\right\} v(x)=g_{2}(x, u(x), v(x))
\end{array}\right.
$$

where both $g_{1}\left(x, y_{1}, y_{2}\right)$ and $g_{2}\left(x, y_{1}, y_{2}\right)$ are mappings from $\Omega \times R \times R$ to $R$, and all coefficient functions $\mu_{i j}(x)$ are Lebesgue integrable and bounded on $\Omega$. Equations (1)-(3) are new in the present studies, and have never been investigated before.

Clearly, Equation (1) turns out to be:

$$
u(x)-c I^{\alpha_{11}} u(x)=f(x), \quad \alpha_{11}>0
$$

if $n=m=1$ and $\lambda_{11}(x)=-c$ (constant). Equation (4) is obviously the classical Abel's integral equation of the second kind. In 1930, Hille and Tamarkin [2] derived its solution as

$$
u(x)=f(x)+c \int_{0}^{x}(x-\tau)^{\alpha_{11}-1} E_{\alpha_{11}, \alpha_{11}}\left(c(x-\tau)^{\alpha_{11}}\right) f(\tau) d \tau,
$$

where:

$$
E_{\alpha, \beta}(z)=\sum_{j=0}^{\infty} \frac{z^{j}}{\Gamma(\alpha j+\beta)}, \quad \alpha, \beta>0
$$

is the Mittag-Leffler function.

There have been many analytic and numerical studies on Abel's integral equation of the second kind, including its variants and generalizations in distribution [3-11]. Cameron and McKee [12] investigated the following Abel's integral equation of the second kind, numerically based on the construction and convergence analysis of the high-order product integral:

$$
u(x)+\int_{0}^{x}(x-s)^{-\alpha} k(x, s, u(s)) d s=f(x),
$$

where $u(x)$ is the unknown function defined on the interval $0 \leq x \leq T<\infty$ and the kernel $k(x, s, u(s))$ is Lipschitz continuous in its third variable. Pskhu [13] constructed an explicit solution for the generalized Abel's integral equation with constant coefficients $c_{k}$ for $k=1,2, \cdots, n$ :

$$
u(x)-\sum_{k=1}^{n} c_{k} I_{k}^{\alpha_{k}} u(x)=f(x), \quad \alpha_{k}>0, \quad x \in \Omega,
$$

using the Wright function:

$$
\phi(\alpha, \beta ; z)=\sum_{j=0}^{\infty} \frac{z^{j}}{j ! \Gamma(\alpha j+\beta)^{\prime}}, \quad \alpha>-1,
$$


and convolution. Evidently, Equation (5) is a special case of our Equation (1) for particular values of $m, \lambda_{i j}(x)$ and $\alpha_{i j}$. In 2019, Li and Plowman [14] derived a convergent solution for the following Abel's integral equation:

$$
u(x)-\left(a_{1}(x) I_{1}^{\alpha_{1}}\right) \cdots\left(a_{n}(x) I_{n}^{\alpha_{n}}\right) u(x)=f(x), \quad x \in \Omega,
$$

based on Babenko's approach in the space $L(\Omega)$. Obviously, Equation (6) is also a particular case of Equation (1) with $m=1, \lambda_{11}(x)=-a_{1}(x), \lambda_{21}(x)=a_{2}(x), \cdots, \lambda_{n 1}(x)=a_{n}(x)$, and $\alpha_{i 1}=\alpha_{i}$ for $i=1,2, \cdots, n$.

In a wide range of scientific and engineering problems, the existence of a solution to an integral equation is equivalent to the existence of a fixed point for a suitable and welldefined mapping on spaces under consideration. Fixed points are therefore essential tools in studying integral equations or systems arising from the real world. Banach's contractive principle provides a general condition ensuring that, if it is satisfied, the iteration of the mapping produces a fixed point [15].

Babenko's approach [16] is a very useful method in solving differential and integral equations, which treat differential or integral operators like variables. The method itself is similar to the Laplace transform when dealing with differential or integral equations with constant coefficients, but it also works for certain equations with distributions, such as $x_{+}^{-1.5}$ and $\delta^{(0.5)}(x)$, whose Laplace transforms do not exist in the classical sense [6,8]. As an example, we are going to solve Equation (4) using this technique. Clearly:

$$
u(x)-c I^{\alpha_{11}} u(x)=\left(1-c I^{\alpha_{11}}\right) u(x)=f(x) .
$$

Informally:

$$
\begin{aligned}
u(x) & =\left(1-c I^{\alpha_{11}}\right)^{-1} f(x)=\sum_{k=0}^{\infty}\left(c I^{\alpha_{11}}\right)^{k} f(x)=\sum_{k=0}^{\infty} c^{k} I^{\alpha_{11} k} f(x) \\
& =f(x)+\sum_{k=0}^{\infty} c^{k+1} I^{\alpha_{11} k+\alpha_{11}} f(x) \\
& =f(x)+c \sum_{k=0}^{\infty} \frac{c^{k}}{\Gamma\left(\alpha_{11} k+\alpha_{11}\right)} \int_{0}^{x}(x-\tau)^{\alpha_{11} k+\alpha_{11}-1} f(\tau) d \tau \\
& =f(x)+c \int_{0}^{x}(x-\tau)^{\alpha_{11}-1} \sum_{k=0}^{\infty} \frac{c^{k}(x-\tau)^{\alpha_{11} k}}{\Gamma\left(\alpha_{11} k+\alpha_{11}\right)} f(\tau) d \tau \\
& =f(x)+c \int_{0}^{x}(x-\tau)^{\alpha_{11}-1} E_{\alpha_{11}, \alpha_{11}}\left(c(x-\tau)^{\alpha_{11}}\right) f(\tau) d \tau,
\end{aligned}
$$

which coincides with Hille and Tamarkin's result provided above.

\section{The Main Results}

In this section, we are going to present our main outcomes with several examples for the illustration of the key theorems.

Theorem 1. Assume that $f \in L(\Omega), \alpha_{i j} \geq 0$, and $\lambda_{i j}(x)$ is Lebesgue integrable and bounded on $\Omega$ for all $i=1,2, \cdots, n$ and $j=1,2, \cdots, m$. In addition, there exists $1 \leq i \leq n$ such that:

$$
\alpha=\min \left\{\alpha_{i 1}, \cdots, \alpha_{i m}\right\} \geq 1 .
$$

Then, Equation (1) has a unique solution in the space $L(\Omega)$ :

$$
\begin{aligned}
u(x)= & \sum_{k=0}^{\infty}(-1)^{k} \sum_{k_{1}+\cdots+k_{m}=k}\left(\begin{array}{c}
k \\
k_{1}, \cdots, k_{m}
\end{array}\right) \\
& \left(\lambda_{11}(x) I_{1}^{\alpha_{11}} \cdots \lambda_{n 1}(x) I_{n}^{\alpha_{n 1}}\right)^{k_{1}} \cdots\left(\lambda_{1 m}(x) I_{1}^{\alpha_{1 m}} \cdots \lambda_{n m}(x) I_{n}^{\alpha_{n m}}\right)^{k_{m}} f(x) .
\end{aligned}
$$


Proof. Equation (1) turns out to be:

$$
\left(1+\sum_{j=1}^{m}\left\{\lambda_{1 j}(x) I_{1}^{\alpha_{1 j}}\right\}\left\{\lambda_{2 j}(x) I_{2}^{\alpha_{2 j}}\right\} \cdots\left\{\lambda_{n j}(x) I_{n}^{\alpha_{n j}}\right\}\right) u(x)=f(x) .
$$

Thus, by Babenko's approach:

$$
\begin{aligned}
u(x)= & \left(1+\sum_{j=1}^{m}\left\{\lambda_{1 j}(x) I_{1}^{\alpha_{1 j}}\right\}\left\{\lambda_{2 j}(x) I_{2}^{\alpha_{2 j}}\right\} \cdots\left\{\lambda_{n j}(x) I_{n}^{\alpha_{n j}}\right\}\right)^{-1} f(x) \\
= & \sum_{k=0}^{\infty}(-1)^{k}\left(\sum_{j=1}^{m}\left\{\lambda_{1 j}(x) I_{1}^{\alpha_{1 j}}\right\}\left\{\lambda_{2 j}(x) I_{2}^{\alpha_{2 j}}\right\} \cdots\left\{\lambda_{n j}(x) I_{n}^{\alpha_{n j}}\right\}\right)^{k} f(x) \\
= & \sum_{k=0}^{\infty}(-1)^{k} \sum_{k_{1}+\cdots+k_{m}=k}\left(\begin{array}{c}
k \\
k_{1}, \cdots, k_{m}
\end{array}\right) \\
& \left(\lambda_{11}(x) I_{1}^{\alpha_{11}} \cdots \lambda_{n 1}(x) I_{n}^{\alpha_{n 1}}\right)^{k_{1}} \cdots\left(\lambda_{1 m}(x) I_{1}^{\alpha_{1 m}} \cdots \lambda_{n m}(x) I_{n}^{\alpha_{n m}}\right)^{k_{m}} f(x) .
\end{aligned}
$$

Obviously, there exists $M>0$ such that:

$$
\sup _{x \in \Omega}\left|\lambda_{i j}(x)\right| \leq M
$$

for all $i=1,2, \cdots, n$ and $j=1,2, \cdots m$.

Let:

$$
\omega=\max \left\{\Omega_{1}, \Omega_{2}, \cdots, \Omega_{n}\right\},
$$

and:

$$
\Phi_{i, \alpha_{i j}}(x)=\frac{\left(x_{i}\right)_{+}^{\alpha_{i j}-1}}{\Gamma\left(\alpha_{i j}\right)} .
$$

Then, it follows from reference [17] that:

$$
\begin{aligned}
\left\|I_{i}^{\alpha_{i j}}\right\| & =\sup _{\|g\| \leq 1}\left\|I_{i}^{\alpha_{i j}} g\right\|=\sup _{\|g\| \leq 1}\left\|\Phi_{i, \alpha_{i j}} * g\right\| \leq \sup _{\|g\| \leq 1}\left\|\Phi_{i, \alpha_{i j}}\right\|\|g\| \leq\left\|\Phi_{i, \alpha_{i j}}\right\| \\
& =\int_{\Omega} \frac{\left(x_{i}\right)_{+}^{\alpha_{i j}-1}}{\Gamma\left(\alpha_{i j}\right)} d x_{1} \cdots d x_{n} \\
& =\Omega_{1} \cdots \Omega_{i-1} \frac{\Omega_{i}^{\alpha_{i j}}}{\Gamma\left(\alpha_{i j}+1\right)} \Omega_{i+1} \cdots \Omega_{n} \leq \omega^{n-1} \frac{\omega^{\alpha_{i j}}}{\Gamma\left(\alpha_{i j}+1\right)} .
\end{aligned}
$$

Therefore:

$$
\begin{aligned}
u(x) \leq & \sum_{k=0}^{\infty}\left(M^{n}\right)^{k} \sum_{k_{1}+\cdots+k_{m}=k}\left(\begin{array}{c}
k \\
k_{1}, \cdots, k_{m}
\end{array}\right)\left\|I_{1}^{\alpha_{11} k_{1}+\cdots+\alpha_{1 m} k_{m}}\right\| \ldots \\
& \left\|I_{n}^{\alpha_{n 1} k_{1}+\cdots+\alpha_{n m} k_{m}}\right\|\|f\| .
\end{aligned}
$$

Clearly:

$$
\begin{aligned}
& \left\|I_{1}^{\alpha_{11} k_{1}+\cdots+\alpha_{1 m} k_{m}}\right\| \leq \omega^{n-1} \frac{\omega^{\alpha_{11} k_{1}+\cdots+\alpha_{1 m} k_{m}}}{\Gamma\left(\alpha_{11} k_{1}+\cdots+\alpha_{1 m} k_{m}+1\right)}, \\
& \cdots, \\
& \left\|I_{n}^{\alpha_{n 1} k_{1}+\cdots+\alpha_{n m} k_{m}}\right\| \leq \omega^{n-1} \frac{\omega^{\alpha_{n 1} k_{1}+\cdots+\alpha_{n m} k_{m}}}{\Gamma\left(\alpha_{n 1} k_{1}+\cdots+\alpha_{n m} k_{m}+1\right)} .
\end{aligned}
$$


Since there exists $1 \leq i \leq n$ such that:

$$
\alpha=\min \left\{\alpha_{i 1}, \cdots, \alpha_{i m}\right\} \geq 1,
$$

which infers that:

$$
\Gamma\left(\alpha_{i 1} k_{1}+\cdots+\alpha_{i m} k_{m}+1\right) \geq \Gamma(\alpha k+1)
$$

for all $k=0,1, \cdots$ by noting that $\Gamma(x+1)$ is an increasing function if $x \geq 1$. Furthermore:

$$
\Gamma\left(\alpha_{s 1} k_{1}+\cdots+\alpha_{s m} k_{m}+1\right) \geq \frac{4}{5}
$$

for $s=1,2, \cdots, i-1, i+1, \cdots, n$ and $k=0,1, \cdots$, since $\Gamma(x+1) \geq 4 / 5$ for all $x \geq 0$. Let:

$$
W=\max _{1 \leq i \leq n, 1 \leq j \leq m}\left\{\omega^{\alpha_{i j}}\right\} .
$$

Applying the identity:

$$
\sum_{k_{1}+k_{2}+\cdots+k_{m}=k}\left(\begin{array}{c}
k \\
k_{1}, k_{2}, \cdots, k_{m}
\end{array}\right)=m^{k}
$$

we derive that:

$$
\begin{aligned}
\|u(x)\| & \leq \omega^{n^{2}-n}\left(\frac{5}{4}\right)^{n-1}\|f\| \sum_{k=0}^{\infty} \frac{\left(M^{n} m W^{n}\right)^{k}}{\Gamma(\alpha k+1)} \\
& =\omega^{n^{2}-n}\left(\frac{5}{4}\right)^{n-1}\|f\| E_{\alpha, 1}\left(M^{n} m W^{n}\right)<\infty .
\end{aligned}
$$

We still need to show that Equation (7) is a solution of Equation (1). Indeed:

$$
\begin{aligned}
& \sum_{k=0}^{\infty}(-1)^{k}\left(\sum_{j=1}^{m}\left\{\lambda_{1 j}(x) I_{1}^{\alpha_{1 j}}\right\}\left\{\lambda_{2 j}(x) I_{2}^{\alpha_{2 j}}\right\} \cdots\left\{\lambda_{n j}(x) I_{n}^{\alpha_{n j}}\right\}\right)^{k} f(x) \\
& =f(x)+\sum_{k=1}^{\infty}(-1)^{k}\left(\sum_{j=1}^{m}\left\{\lambda_{1 j}(x) I_{1}^{\alpha_{1 j}}\right\}\left\{\lambda_{2 j}(x) I_{2}^{\alpha_{2 j}}\right\} \cdots\left\{\lambda_{n j}(x) I_{n}^{\alpha_{n j}}\right\}\right)^{k} f(x),
\end{aligned}
$$

and:

$$
\begin{aligned}
& \sum_{k=1}^{\infty}(-1)^{k}\left(\sum_{j=1}^{m}\left\{\lambda_{1 j}(x) I_{1}^{\alpha_{1 j}}\right\}\left\{\lambda_{2 j}(x) I_{2}^{\alpha_{2 j}}\right\} \cdots\left\{\lambda_{n j}(x) I_{n}^{\alpha_{n j}}\right\}\right)^{k} f(x) \\
& +\sum_{k=0}^{\infty}(-1)^{k}\left(\sum_{j=1}^{m}\left\{\lambda_{1 j}(x) I_{1}^{\alpha_{1 j}}\right\}\left\{\lambda_{2 j}(x) I_{2}^{\alpha_{2 j}}\right\} \cdots\left\{\lambda_{n j}(x) I_{n}^{\alpha_{n j}}\right\}\right) . \\
& \left(\sum_{j=1}^{m}\left\{\lambda_{1 j}(x) I_{1}^{\alpha_{1 j}}\right\}\left\{\lambda_{2 j}(x) I_{2}^{\alpha_{2 j}}\right\} \cdots\left\{\lambda_{n j}(x) I_{n}^{\alpha_{n j}}\right\}\right)^{k} f(x)=0,
\end{aligned}
$$

by noting that all of the above series are uniformly and absolutely convergent in the space $L(\Omega)$ due to inequality (8).

Evidently, the uniqueness immediately follows from the fact that the homogeneous integral equation:

$$
u(x)+\sum_{j=1}^{m}\left\{\lambda_{1 j}(x) I_{1}^{\alpha_{1 j}}\right\}\left\{\lambda_{2 j}(x) I_{2}^{\alpha_{2 j}}\right\} \cdots\left\{\lambda_{n j}(x) I_{n}^{\alpha_{n j}}\right\} u(x)=0
$$

only has solution zero by Babenko's method. This completes the proof of Theorem 2. 
Remark 1. Note that $\Gamma(x+1)$ is not a monotone increasing function on $[0,1]$ since $\Gamma(1)=1$, $\Gamma(1.5)=\sqrt{\pi} / 2$ and $\Gamma(2)=1$.

Example 1. Abel's integral equation:

$$
u\left(x_{1}, x_{2}\right)+x_{1} I_{1}^{0.5} x_{1}^{2} I_{2} u\left(x_{1}, x_{2}\right)+x_{2}^{0.1} I_{2}^{1.5} u\left(x_{1}, x_{2}\right)=1
$$

has the following convergent solution in $L(\Omega)$ :

$$
u(x)=1+\sum_{k=1}^{\infty}(-1)^{k} \sum_{j=0}^{k}\left(\begin{array}{l}
k \\
j
\end{array}\right) B_{j} A_{k-j} \Phi_{1,1+3.5 j} \Phi_{2,1+j+(k-j) 1.6}
$$

where the coefficients $B_{j}$ and $A_{k-j}$ are given below.

Proof. Clearly:

$$
\alpha=\min \left\{\alpha_{21}, \alpha_{22}\right\}=\min \{1,1.5\}=1,
$$

and functions $x_{1}, x_{1}^{2}$ and $x_{2}^{0.1}$ are Lebesgue integrable and bounded on $\Omega$. By Theorem 1 :

$$
\begin{aligned}
u(x) & =1+\sum_{k=1}^{\infty}(-1)^{k}\left(x_{1} I_{1}^{0.5} x_{1}^{2} I_{2}+x_{2}^{0.1} I_{2}^{1.5}\right)^{k} 1 \\
& =1+\sum_{k=1}^{\infty}(-1)^{k} \sum_{j=0}^{k}\left(\begin{array}{l}
k \\
j
\end{array}\right)\left(x_{1} I_{1}^{0.5} x_{1}^{2} I_{2}\right)^{j}\left(x_{2}^{0.1} I_{2}^{1.5}\right)^{k-j} 1 .
\end{aligned}
$$

Obviously:

$$
\begin{aligned}
& \left(x_{2}^{0.1} I_{2}^{1.5}\right)^{0} 1=1, \\
& \left(x_{2}^{0.1} I_{2}^{1.5}\right) 1=\left(x_{2}^{0.1} I_{2}^{1.5}\right) \Phi_{2,1}=x_{2}^{0.1}\left(\Phi_{2,1.5} * \Phi_{2,1}\right)=x_{2}^{0.1} \Phi_{2,2.5}=\frac{\left(x_{2}\right)_{+}^{1.6}}{\Gamma(2.5)} \\
& =\frac{\Gamma(2.6)}{\Gamma(2.5)} \Phi_{2,2.6} \\
& \left(x_{2}^{0.1} I_{2}^{1.5}\right)^{2} 1=\left(x_{2}^{0.1} I_{2}^{1.5}\right) \frac{\Gamma(2.6)}{\Gamma(2.5)} \Phi_{2,2.6}=\frac{\Gamma(2.6) \Gamma(4.2)}{\Gamma(2.5) \Gamma(4.1)} \Phi_{2,4.2} \\
& \left(x_{2}^{0.1} I_{2}^{1.5}\right)^{3} 1=\left(x_{2}^{0.1} I_{2}^{1.5}\right) \frac{\Gamma(2.6) \Gamma(4.2)}{\Gamma(2.5) \Gamma(4.1)} \Phi_{2,4.2}=\frac{\Gamma(2.6) \Gamma(4.2) \Gamma(5.8)}{\Gamma(2.5) \Gamma(4.1) \Gamma(5.7)} \Phi_{2,5.8} \\
& \ldots, \\
& \left(x_{2}^{0.1} I_{2}^{1.5}\right)^{k-j} 1=\frac{\Gamma(2.6) \Gamma(4.2) \cdots \Gamma(1+(k-j) 1.6)}{\Gamma(2.5) \Gamma(4.1) \cdots \Gamma(0.9+(k-j) 1.6)} \Phi_{2,1+(k-j) 1.6} \\
& I_{2}^{j}\left(x_{2}^{0.1} I_{2}^{1.5}\right)^{k-j} 1=\frac{\Gamma(2.6) \Gamma(4.2) \cdots \Gamma(1+(k-j) 1.6)}{\Gamma(2.5) \Gamma(4.1) \cdots \Gamma(0.9+(k-j) 1.6)} \Phi_{2,1+j+(k-j) 1.6}, \\
& =A_{k-j} \Phi_{2,1+j+(k-j) 1.6 \prime}
\end{aligned}
$$

for $k-j=1,2, \cdots$, and:

$$
A_{k-j}= \begin{cases}\frac{\Gamma(2.6) \Gamma(4.2) \cdots \Gamma(1+(k-j) 1.6)}{\Gamma(2.5) \Gamma(4.1) \cdots \Gamma(0.9+(k-j) 1.6)} & \text { if } k-j \geq 1 \\ 1 & \text { if } k-j=0\end{cases}
$$


On the other hand:

$$
\begin{aligned}
& \left(x_{1} I_{1}^{0.5} x_{1}^{2}\right)^{0}=1, \\
& x_{1} I_{1}^{0.5} x_{1}^{2}=x_{1} \Phi_{1,0.5} * \Gamma(3) \Phi_{1,3}=\frac{\Gamma(3) \Gamma(4.5)}{\Gamma(3.5)} \Phi_{1,4.5}, \\
& \left(x_{1} I_{1}^{0.5} x_{1}^{2}\right)^{2}=\frac{\Gamma(3) \Gamma(4.5)}{\Gamma(3.5)}\left(x_{1} I_{1}^{0.5} x_{1}^{2}\right) \Phi_{1,4.5}=\frac{\Gamma(3) \Gamma(6.5) \Gamma(8)}{\Gamma(3.5) \Gamma(7)} \Phi_{1,8}, \\
& \left(x_{1} I_{1}^{0.5} x_{1}^{2}\right)^{3}=\frac{\Gamma(3) \Gamma(6.5) \Gamma(10) \Gamma(11.5)}{\Gamma(3.5) \Gamma(7) \Gamma(10.5)} \Phi_{1,11.5} \\
& \ldots, \\
& \left(x_{1} I_{1}^{0.5} x_{1}^{2}\right)^{j}=\frac{\Gamma(3) \Gamma(6.5) \cdots \Gamma(3+3.5(j-1)) \Gamma(1+3.5 j)}{\Gamma(3.5) \Gamma(7) \cdots \Gamma(3.5 j)} \Phi_{1,1+3.5 j,} \\
& =B_{j} \Phi_{1,1+3.5 j},
\end{aligned}
$$

for $j=1,2, \cdots$, and:

$$
B_{j}= \begin{cases}\frac{\Gamma(3) \Gamma(6.5) \cdots \Gamma(3+3.5(j-1)) \Gamma(1+3.5 j)}{\Gamma(3.5) \Gamma(7) \cdots \Gamma(3.5 j)} & \text { if } j \geq 1, \\ 1 & \text { if } j=0 .\end{cases}
$$

Therefore:

$$
u(x)=1+\sum_{k=1}^{\infty}(-1)^{k} \sum_{j=0}^{k}\left(\begin{array}{l}
k \\
j
\end{array}\right) B_{j} A_{k-j} \Phi_{1,1+3.5 j} \Phi_{2,1+j+(k-j) 1.6} .
$$

This completes the proof of Example 1.

Using Banach's fixed point theorem, we are now ready to show the uniqueness of solutions in $L(\Omega)$ for Equation (2).

Theorem 2. Suppose that $\alpha_{i j} \geq 0$, and $\lambda_{i j}(x)$ is Lebesgue integrable and bounded on $\Omega$ for $i=1,2, \cdots, n$ and $j=1,2, \cdots, m$, and there exists $1 \leq i \leq n$ such that:

$$
\alpha=\min \left\{\alpha_{i 1}, \cdots, \alpha_{i m}\right\} \geq 1 .
$$

Let $g(x, y)$ be defined on $\Omega \times R$ satisfying:

$$
\left|g\left(x, y_{1}\right)-g\left(x, y_{2}\right)\right| \leq C\left|y_{1}-y_{2}\right|,
$$

and $g(x, 0) \in L(\Omega)$. Furthermore, assume that:

$$
q=C \omega^{n^{2}-n}\left(\frac{5}{4}\right)^{n-1} E_{\alpha, 1}\left(M^{n} m W^{n}\right)<1
$$

where $\omega, M, W$ are given in Theorem 1 as

$$
\begin{aligned}
& \omega=\max \left\{\Omega_{1}, \Omega_{2}, \cdots, \Omega_{n}\right\}, \quad \sup _{x \in \Omega}\left|\lambda_{i j}(x)\right| \leq M, \\
& W=\max _{1 \leq i \leq n, 1 \leq j \leq m}\left\{\omega^{\alpha_{i j}}\right\} .
\end{aligned}
$$

Then, Equation (2) has a unique solution in $L(\Omega)$.

Proof. Let $u \in L(\Omega)$. We first show that $g(x, u(x)) \in L(\Omega)$. Indeed:

$$
\begin{aligned}
|g(x, u(x))| & =|g(x, u)-g(x, 0)+g(x, 0)| \leq|g(x, u)-g(x, 0)|+|g(x, 0)| \\
& \leq C|u|+|g(x, 0)|
\end{aligned}
$$


which implies that:

$$
\int_{\Omega}|g(x, u(x))| d x \leq C \int_{\Omega}|u| d x+\int_{\Omega}|g(x, 0)| d x<\infty .
$$

Define a nonlinear mapping $T$ on $L(\Omega)$ by

$$
\begin{aligned}
T(u)= & \sum_{k=0}^{\infty}(-1)^{k} \sum_{k_{1}+\cdots+k_{m}=k}\left(\begin{array}{c}
k \\
k_{1}, \cdots, k_{m}
\end{array}\right) \\
& \left(\lambda_{11}(x) I_{1}^{\alpha_{11}} \cdots \lambda_{n 1}(x) I_{n}^{\alpha_{n 1}}\right)^{k_{1}} \cdots\left(\lambda_{1 m}(x) I_{1}^{\alpha_{1 m}} \cdots \lambda_{n m}(x) I_{n}^{\alpha_{n m}}\right)^{k_{m}} g(x, u) .
\end{aligned}
$$

Clearly:

$$
\|T(u)\| \leq \omega^{n^{2}-n}\left(\frac{5}{4}\right)^{n-1} E_{\alpha, 1}\left(M^{n} m W^{n}\right) \int_{\Omega}|g(x, u(x))| d x<\infty .
$$

Thus, $T$ is a mapping from $L(\Omega)$ to $L(\Omega)$. We now need to show that $T$ is a contractive mapping. In fact:

$$
\begin{aligned}
\|T(u)-T(v)\| & \leq \omega^{n^{2}-n}\left(\frac{5}{4}\right)^{n-1} E_{\alpha, 1}\left(M^{n} m W^{n}\right) \int_{\Omega}|g(x, u)-g(x, v)| d x \\
& \leq C \omega^{n^{2}-n}\left(\frac{5}{4}\right)^{n-1} E_{\alpha, 1}\left(M^{n} m W^{n}\right)\|u-v\|=q\|u-v\|,
\end{aligned}
$$

which claims that $T$ is contractive since $q<1$. This completes the proof of Theorem 2 .

Example 2. Let $\Omega=[0,1] \times[0,1] \times[0,1]$. Then, the generalized Abel's integral equation:

$$
\begin{aligned}
& u\left(x_{1}, x_{2}, x_{3}\right)+x_{1} I_{1}^{0.5} \sin \left(x_{1} x_{2}\right) I_{2}^{1.7} \cos \left(x_{3}^{2}+1\right) I_{3}^{0.2} u\left(x_{1}, x_{2}, x_{3}\right) \\
& -x_{1}^{3} I_{1}^{1.5} x_{2}^{0.5} I_{2} u\left(x_{1}, x_{2}, x_{3}\right)=\frac{1}{7 \pi} \arctan \left(x_{1}^{2}+x_{2}^{2}\right) \cos \left(u\left(x_{1}, x_{2}, x_{3}\right)+1\right)
\end{aligned}
$$

has a unique solution in $L(\Omega)$.

Proof. Clearly, $m=2, \omega=\max \{1,1,1\}=1, W=\max _{1 \leq i \leq n, 1 \leq j \leq m}\left\{\omega^{\alpha_{i j}}\right\}=1$, and:

$$
\alpha=\min \{1.7,1\}=1 \text {. }
$$

Furthermore:

$$
\begin{aligned}
& \left|x_{1}\right| \leq 1, \quad\left|\sin \left(x_{1} x_{2}\right)\right| \leq 1, \quad\left|\cos \left(x_{3}^{2}+1\right)\right| \leq 1 \\
& \left|-x_{1}^{3}\right| \leq 1, \quad\left|x_{2}^{0.5}\right| \leq 1,
\end{aligned}
$$

on $\Omega$. Therefore, $M=1$. Obviously:

$$
g\left(x_{1}, x_{2}, x_{3}, y\right)=\frac{1}{7 \pi} \arctan \left(x_{1}^{2}+x_{2}^{2}\right) \cos (y+1),
$$

$g\left(x_{1}, x_{2}, x_{3}, 0\right) \in L(\Omega)$, and:

$$
\left|g\left(x_{1}, x_{2}, x_{3}, y_{1}\right)-g\left(x_{1}, x_{2}, x_{3}, y_{2}\right)\right| \leq \frac{1}{7 \pi} \frac{\pi}{2}\left|y_{1}-y_{2}\right|=\frac{1}{14}\left|y_{1}-y_{2}\right| .
$$


It remains to compute the value of $q$ :

$$
\begin{aligned}
q & =\frac{1}{14}\left(\frac{5}{4}\right)^{3-1} E_{1,1}(2)=\frac{25}{224} \sum_{j=0}^{\infty} \frac{2^{j}}{j !} \\
& =\frac{25}{224}\left(1+2+\frac{2 \cdot 2}{1 \cdot 2}+\frac{2 \cdot 2 \cdot 2}{1 \cdot 2 \cdot 3}+\frac{2 \cdot 2 \cdot 2 \cdot 2}{1 \cdot 2 \cdot 3 \cdot 4}+\frac{2 \cdot 2 \cdot 2 \cdot 2 \cdot 2}{1 \cdot 2 \cdot 3 \cdot 4 \cdot 5}+\cdots\right) \\
& \leq \frac{25}{224}\left(1+2+2+\left(\frac{1}{3}+\left(\frac{2}{3}\right)^{0}\right)+\left(\frac{2}{3}\right)^{1}+\left(\frac{2}{3}\right)^{2}+\cdots\right)=\frac{625}{672}<1 .
\end{aligned}
$$

By Theorem 2, Equation (9) has a unique solution in $L(\Omega)$. This completes the proof of Example 2.

Finally, we study the uniqueness of solutions of in-symmetry system (3) in the product space $L(\Omega) \times L(\Omega)$.

Theorem 3. Suppose that $\alpha_{i j} \geq 0, \beta_{i j} \geq 0$, and $\lambda_{i j}(x), \mu_{i j}(x)$ are Lebesgue integrable and bounded on $\Omega$ for $i=1,2, \cdots, n$ and $j=1,2, \cdots, m$, and there exists $1 \leq i_{1}, i_{2} \leq n$ such that:

$$
\begin{aligned}
& \alpha=\min \left\{\alpha_{i_{1} 1}, \cdots, \alpha_{i_{1} m}\right\} \geq 1, \\
& \beta=\min \left\{\beta_{i_{2} 1}, \cdots, \beta_{i_{2} m}\right\} \geq 1
\end{aligned}
$$

Let $g_{1}\left(x, y_{1}, y_{2}\right)$ and $g_{2}\left(x, y_{1}, y_{2}\right)$ be defined on $\Omega \times R \times R$ satisfying:

$$
\begin{aligned}
& \left|g_{1}\left(x, y_{1}, y_{2}\right)-g_{1}\left(x, z_{1}, z_{2}\right)\right| \leq C_{1}\left|y_{1}-z_{1}\right|+C_{2}\left|y_{2}-z_{2}\right|, \\
& \left|g_{2}\left(x, s_{1}, s_{2}\right)-g_{2}\left(x, t_{1}, t_{2}\right)\right| \leq C_{3}\left|s_{1}-t_{1}\right|+C_{4}\left|s_{2}-t_{2}\right|,
\end{aligned}
$$

and $g_{1}(x, 0,0), g_{2}(x, 0,0) \in L(\Omega)$. Furthermore, assume that:

$$
\begin{aligned}
q= & \max \left\{C_{1}, C_{2}\right\} \omega^{n^{2}-n}\left(\frac{5}{4}\right)^{n-1} E_{\alpha, 1}\left(M_{1}^{n} m W_{1}^{n}\right) \\
& +\max \left\{C_{3}, C_{4}\right\} \omega^{n^{2}-n}\left(\frac{5}{4}\right)^{n-1} E_{\beta, 1}\left(M_{2}^{n} m W_{2}^{n}\right)<1,
\end{aligned}
$$

where $M_{1}, W_{1}$ and $M_{2}, W_{2}$ are given as

$$
\begin{aligned}
& \sup _{x \in \Omega}\left|\lambda_{i j}(x)\right| \leq M_{1}, \quad W_{1}=\max _{1 \leq i \leq n, 1 \leq j \leq m}\left\{\omega^{\alpha_{i j}}\right\}, \\
& \sup _{x \in \Omega}\left|\mu_{i j}(x)\right| \leq M_{2}, \quad W_{2}=\max _{1 \leq i \leq n, 1 \leq j \leq m}\left\{\omega^{\beta_{i j}}\right\} .
\end{aligned}
$$

Then, the in-symmetry system (3) has a unique solution in $L(\Omega) \times L(\Omega)$.

Proof. Let $u$, vs. $\in L(\Omega)$. We first show that $g_{1}(x, u(x), v(x)) \in L(\Omega)$. Indeed:

$$
\begin{aligned}
& \left|g_{1}(x, u(x), v(x))\right|=\left|g_{1}(x, u, v)-g_{1}(x, 0,0)+g_{1}(x, 0,0)\right| \\
& \leq\left|g_{1}(x, u, v)-g_{1}(x, 0,0)\right|+\left|g_{1}(x, 0,0)\right| \\
& \leq C_{1}|u|+C_{2}|v|+\left|g_{1}(x, 0,0)\right|
\end{aligned}
$$

which implies that:

$$
\int_{\Omega}\left|g_{1}(x, u(x), v(x))\right| d x \leq C_{1} \int_{\Omega}|u| d x+C_{2} \int_{\Omega}|v| d x+\int_{\Omega}\left|g_{1}(x, 0,0)\right| d x<\infty .
$$

Similarly, $g_{2}(x, u(x), v(x)) \in L(\Omega)$. 
Define a mapping $T$ on $L(\Omega) \times L(\Omega)$ as

$$
T(u, v)=\left(T_{1}(u, v), T_{2}(u, v)\right),
$$

where:

$$
\|T(u, v)\|=\left\|T_{1}(u, v)\right\|+\left\|T_{2}(u, v)\right\|,
$$

and:

$$
\begin{aligned}
T_{1}(u, v)= & \sum_{k=0}^{\infty}(-1)^{k} \sum_{k_{1}+\cdots+k_{m}=k}\left(\begin{array}{c}
k \\
k_{1}, \cdots, k_{m}
\end{array}\right) \\
& \left(\lambda_{11}(x) I_{1}^{\alpha_{11}} \cdots \lambda_{n 1}(x) I_{n}^{\alpha_{n 1}}\right)^{k_{1}} \cdots\left(\lambda_{1 m}(x) I_{1}^{\alpha_{1 m}} \cdots \lambda_{n m}(x) I_{n}^{\alpha_{n m}}\right)^{k_{m}} g_{1}(x, u, v),
\end{aligned}
$$

and symmetrically:

$$
\begin{aligned}
T_{2}(u, v)= & \sum_{k=0}^{\infty}(-1)^{k} \sum_{k_{1}+\cdots+k_{m}=k}\left(\begin{array}{c}
k \\
k_{1}, \cdots, k_{m}
\end{array}\right) \\
& \left(\mu_{11}(x) I_{1}^{\beta_{11}} \cdots \mu_{n 1}(x) I_{n}^{\beta_{n 1}}\right)^{k_{1}} \cdots\left(\mu_{1 m}(x) I_{1}^{\beta_{1 m}} \cdots \mu_{n m}(x) I_{n}^{\beta_{n m}}\right)^{k_{m}} g_{2}(x, u, v) .
\end{aligned}
$$

By inequality (8):

$$
\begin{aligned}
& \left\|T_{1}(u, v)\right\| \leq \omega^{n^{2}-n}\left(\frac{5}{4}\right)^{n-1} E_{\alpha, 1}\left(M_{1}^{n} m W_{1}^{n}\right) \int_{\Omega}\left|g_{1}(x, u(x), v(x))\right| d x<\infty, \\
& \left\|T_{2}(u, v)\right\| \leq \omega^{n^{2}-n}\left(\frac{5}{4}\right)^{n-1} E_{\beta, 1}\left(M_{2}^{n} m W_{2}^{n}\right) \int_{\Omega}\left|g_{2}(x, u(x), v(x))\right| d x<\infty .
\end{aligned}
$$

Hence, $T$ is a mapping from $L(\Omega) \times L(\Omega)$ to $L(\Omega) \times L(\Omega)$. It remains to show that $T$ is contractive. In fact:

$$
\left\|T\left(u_{1}, v_{1}\right)-T\left(u_{2}, v_{2}\right)\right\|=\left\|T_{1}\left(u_{1}, v_{1}\right)-T_{1}\left(u_{2}, v_{2}\right)\right\|+\left\|T_{2}\left(u_{1}, v_{1}\right)-T\left(u_{2}, v_{2}\right)\right\| .
$$

Clearly:

$$
\begin{aligned}
\left\|T_{1}\left(u_{1}, v_{1}\right)-T_{1}\left(u_{2}, v_{2}\right)\right\| \leq & \max \left\{C_{1}, C_{2}\right\} \omega^{n^{2}-n}\left(\frac{5}{4}\right)^{n-1} E_{\alpha, 1}\left(M_{1}^{n} m W_{1}^{n}\right) . \\
& \left\|\left(u_{1}, v_{1}\right)-\left(u_{2}, v_{2}\right)\right\|,
\end{aligned}
$$

and:

$$
\begin{aligned}
\left\|T_{2}\left(u_{1}, v_{1}\right)-T_{2}\left(u_{2}, v_{2}\right)\right\| \leq & \max \left\{C_{3}, C_{4}\right\} \omega^{n^{2}-n}\left(\frac{5}{4}\right)^{n-1} E_{\beta, 1}\left(M_{2}^{n} m W_{2}^{n}\right) . \\
& \left\|\left(u_{1}, v_{1}\right)-\left(u_{2}, v_{2}\right)\right\| .
\end{aligned}
$$

Thus:

$$
\left\|T\left(u_{1}, v_{1}\right)-T\left(u_{2}, v_{2}\right)\right\| \leq q\left\|\left(u_{1}, v_{1}\right)-\left(u_{2}, v_{2}\right)\right\|,
$$

where:

$$
\begin{aligned}
q= & \max \left\{C_{1}, C_{2}\right\} \omega^{n^{2}-n}\left(\frac{5}{4}\right)^{n-1} E_{\alpha, 1}\left(M_{1}^{n} m W_{1}^{n}\right) \\
& +\max \left\{C_{3}, C_{4}\right\} \omega^{n^{2}-n}\left(\frac{5}{4}\right)^{n-1} E_{\beta, 1}\left(M_{2}^{n} m W_{2}^{n}\right)<1 .
\end{aligned}
$$

This completes the proof of Theorem 3. 


\section{Conclusions}

We studied the uniqueness of the solutions of the nonlinear Abel's integral equations of the second kind with variable coefficients and the in-symmetry system based on Banach's fixed point theorem and Babenko's approach. The results are new in the current works of integral equations, which are not feasible by any integral transforms. We also presented several examples to demonstrate the use of our main theorems via some special functions and convolutions.

Author Contributions: Conceptualization, C.L. and J.B.; methodology, C.L.; software, C.L. and J.B.; validation, C.L. and J.B.; formal analysis, C.L.; investigation, C.L. and J.B.; resources, C.L.; data curation, C.L. and J.B.; writing-original draft preparation, C.L.; writing-review and editing, C.L.; visualization, C.L.; supervision, C.L.; project administration, C.L. and J.B.; funding acquisition, C.L. All authors have read and agreed to the published version of the manuscript.

Funding: This work is supported by the Natural Sciences and Engineering Research Council of Canada (Grant No. 2019-03907).

Institutional Review Board Statement: Not applicable.

Informed Consent Statement: Not applicable.

Data Availability Statement: Not applicable.

Acknowledgments: The authors are grateful to the two reviewers for their careful reading of the paper with productive comments and suggestions.

Conflicts of Interest: The authors declare no conflict of interest.

\section{References}

1. Kilbas, A.A.; Srivastava, H.M.; Trujillo, J.J. Theory and Applications of Fractional Differential Equations; Elsevier: Amsterdam, The Netherlands, 2006

2. Hille, E.; Tamarkin, J.D. On the theory of linear integral equations. Ann. Math. 1930, 31, 479-528. [CrossRef]

3. Evans, G.C. Volterra's integral equation of the second kind, with discontinuous kernel. Trans. Am. Math. Soc. 1910, 11, $393-413$.

4. Evans, G.C. Volterra's integral equation of the second kind, with discontinuous kernel, second paper. Trans. Am. Math. Soc. 1911, 12, 429-472. [CrossRef]

5. Gorenflo, R.; Mainardi, F. Fractional Calculus: Integral and Differential Equations of Fractional Order. In Fractals and Fractional Calculus in Continuum Mechanics; Springer: New York, NY, USA, 1997; pp. 223-276.

6. Podlubny, I. Fractional Differential Equations; Academic Press: New York, NY, USA, 1999.

7. Srivastava, H.M.; Buschman, R.G. Theory and Applications of Convolution Integral Equations; Kluwer Academic Publishers: Dordrecht, The Netherlands; Boston, MA, USA; London, UK, 1992.

8. Li, C.; Clarkson, K. Babenko's approach to Abel's integral equations. Mathematics 2018, 6, 32. [CrossRef]

9. Li, C. Several results of fractional derivatives in $D^{\prime}\left(R_{+}\right)$. Fract. Calc. Appl. Anal. 2015, 18, 192-207. [CrossRef]

10. Samko, S.G.; Kilbas, A.A.; Marichev, O.I. Fractional Integrals and Derivatives: Theory and Applications; Gordon and Breach: New York, NY, USA, 1993.

11. Miller, K.S.; Ross, B. An Introduction to the Fractional Calculus and Fractional Differential Equations; Wiley: New York, NY, USA, 1993.

12. Cameron, R.F.; McKee, S. Product integration methods for second-kind Abel integral equations. J. Comput. Appl. Math. 1984, 11, 1-10. [CrossRef]

13. Pskhu, A. Solution of a multidimensional Abel integral equation of the second kind with partial fractional integrals. Differ. Uravn. 2017, 53, 1195-1199. (In Russian) [CrossRef]

14. Li, C.; Plowman, H. Solutions of the generalized Abel's integral equations of the second kind with variable coefficients. Axioms 2019, 8, 137. [CrossRef]

15. Rudin, W. Principle of Mathematical Analysis, 3rd ed.; McGraw-Hill: New York, NY, USA, 1976.

16. Babenkos, Y.I. Heat and Mass Transfer; Khimiya: Leningrad, Russia, 1986. (In Russian)

17. Barros-Neto, J. An Introduction to the Theory of Distributions; Marcel Dekker, Inc.: New York, NY, USA, 1973. 